\title{
Research on the Mobile Communication Network Optimization Based on the Cloud Computing Model
}

\author{
GUO Yan ${ }^{1, a}$ \\ ${ }^{1}$ Chongqing College Of Electronic Engineering, Chongqing 401331,China \\ a guoyan401331@126.com
}

Keywords: Mobile Communication Network, Optimization, Cloud Computing Model

\begin{abstract}
In order to maximize the use of existing mobile communication network resources and enhance the efficiency of mobile communication network optimization, we introduce the cloud computing model. Combined with the situation of mobile communication network optimization, this paper elaborated the characteristics cloud computing and analysis the possibility and necessity of the cloud computing model in the mobile communication network optimization applications. And combined with the characteristics of cloud computing, this paper proposed a mobile communication network optimization link frame based on cloud computing model.
\end{abstract}

\section{Introduction}

From 1982 GSM (Global System for Mobile Communications) European Telecommunications Standards to 1992 large-scale commercial, and then to 3G (the Third Generation, the third generation mobile communication technology) network to accelerate the deployment of today, the modern wireless communications technology showing the trend of rapid development. Behind the large-scale construction in the mobile communication network must exist depth how to optimize the network, effective use of existing resources and other issues. While mobile operators increasingly attach importance to network optimization problems, but due to operating equipment from different manufacturers, it will face massive equipment how to integrate data from different manufacturers. In addition, the network optimization collecting, analyzing data there is uncertainty, resulting in a complex, cumbersome of network optimization solutions. The emergence of cloud computing optimization provides a new, more desirable approach for mobile communications network.

\section{The Current Situation of Network Optimization}

Since mobile operators use equipment from different manufacturers, large amounts of data while optimizing collection there are too many uncertainties, resulting in network optimization software has the following problems [1]:

Unity of Data Processing. Because operators use different devices, so device data optimization process is also different. Today's software can analyze and process optimization for a performance of the network or only to a certain area of the data. This will inevitably affect the generic network optimization software.

Resource Exclusivity. The current network optimization software generally is a stand-alone environment, resulting in incomplete data optimization, can not achieve a shared resource. So that the operators are required to spend a lot of manpower, material resources to complete optimization.

Limited Data Management. The mobile communication network optimization not only to collect a large amount of communication data, comprising: a user call data, file transfer data, log data, etc., and network optimization requires a lot of experience is required to store a large amount of historical data, including optimization embodiments, optimization log and so on, we need strong management system to complete the classification and management of data. Although optimization software uses a large-capacity database, but the software is running low efficiency, can not meet the requirements [2]. 


\section{The Concept of Cloud Computing.}

Cloud computing is the future of grid computing and the emergence of a new computing model, or a kind of commercial implementations of grid computing. People of different backgrounds to understand cloud computing varies. Cloud computing is a Web-based computing and resource service model, distributed computing, parallel computing, load balancing and so on a new mobile computer-based application technology and network technology combined with new computing technologies. In the network topology is often used to represent the cloud telecommunications network, it was also used to represent abstract internet and the underlying infrastructure. The definition of cloud computing have a variety of views. For in the end what is cloud computing, say, than the statistics down, at least you can find 100 kinds of explanations. Currently, more acceptable definition of cloud computing, deputy director of China Expert Advisory Committee, the Secretary-General Professor Liu Peng given: "Cloud computing is provided through a network of low-cost scalable distributed computing capabilities" can be found through the Internet cloud to provide the latest and most timely service network, which uses a network spread this virtual world, share stores various information resources, can also use the repository to store or calculate formed.

The expert Rajkumar Buyya of Melbourne University defines computer science and software engineering people on the cloud as: "Cloud computing is a composed of a series of interconnected and unified computing resource base in real time and dynamically provide the user as one or more Parallel Distributed Systems virtualized computer resources consisting of these unified computing resource library is based on established between the service providers and consumers to negotiate on the basis of service level agreements. [3]"

Kai-fu Lee believes that: the so-called "cloud computing" is to open standards and service-based, Internet-centric, to provide safe, fast, convenient data storage and network computing services to the Internet this "cloud" has become each users of data center and computing center.

We can see, cloud computing is a revolution - the user simply through the browser can easily access the "cloud" resources through a network cable. It will change the traditional hardware and software used.

\section{The Characteristics of Cloud Computing}

Cloud computing platform not only has the characteristics of clusters and grid computing, and has the following features and functions of their own [4].

Internet-Centric. Cloud computing platform operators to Internet-centric, storage and computing power distributed among the various nodes of the network is connected, thereby weakening the computing capability of the terminal, so that computing architecture of the Internet by the "Server + Client" to "cloud services platform + Client "evolution. Cloud computing operators of the Internet as a center, and that each node in the network chain has two functions, one is storage, one is computing functions, operators will be arranged, in order to reduce the load on the network terminal, so that Internet users In using the Internet, you can more quickly and efficiently get the information they want.

Based on Virtualization. Virtualization is the foundation of cloud computing technology, it underlying hardware, including servers, storage and network equipment virtualized, on top of virtualization technology to create a demand and selected on the basis of a shared resource pool resources to form a service type of scalable IT infrastructure to provide users with the resources to hire IT infrastructure in the form of cloud computing services.

Super Computing Power. Scheduling policy coordination through certain cloud computing model can provide powerful, can compete with supercomputers by combining the computing power of tens of thousands and even millions of ordinary computers, allowing users to complete a single computer can not be completed task.

Cost-Effective Services. Cloud computing to provide users with the most affordable and convenient services, cloud computing, users only need to spend very little money, not even money can use the calculation function before they can not have and have resources from around the world. 
Safe Data Center. Cloud computing provides a secure, reliable data storage center, strict rights management strategies can help users to be assured of the sharing of data with a specified person. Cloud computing has a powerful memory feature that can store large volumes of data and information. However, many users are concerned about cloud storage is safe. In fact, this point the user is totally unnecessary to worry about, because, cloud computing operators have very sound security management measures in this regard, the information stored is limited to users themselves visible, unless the user specifies a friend have access to their own the ability to store information, but only the designated friends can see the information they store.

\section{The Conjunction with Cloud Computing Model and Network Optimization}

In the mobile communication network optimization which based on cloud computing model, the identification system, data storage and cloud resources and improve the environment and personal communications network terminal equipment combined. By a communication network optimization cloud computing service providers to telecommunications service operators, carriers through a unique user name, after obtaining certification, and then access cloud resources through the network, to achieve the desired optimization. Not only have a more powerful and enhances the security of the system, with better prospects for development [5].

As a new, award-winning technology, cloud computing technology, once launched, it has been respected person concerned. Such as: Microsoft is developing a completely out of the desktop operating system of Internet "Midori", IBM's Blue Cloud computing platforms and Amazon's Elastic Compute Cloud. The emergence of cloud computing model for mobile communication network optimization injection of fresh blood, combined with the characteristics of cloud computing and network optimization work status quo, cloud computing mobile communications network will bring the following profound changes.

Change Mode. Under the conventional mobile communication network optimization mode environment, mobile operators have to face a variety of multi-system applications and devices from different manufacturers, each device needs to deal with inconsistent application of control mechanisms and to integrate devices from different manufacturers mass of data . Even with a device inside the integration is not good. In order to enhance the usability of the system, operators and equipment necessary for each application separately requested, occasionally compromise to technical limitations.

The use of cloud computing model, will receive collaborative support of many services. No matter which device vendors, carriers do not care about the details of the integration process, just to enjoy the service. Therefore, network optimization, you can omit the data pretreatment process data and optimize acquisition without integration, network optimization greatly improved work efficiency.

Low Cost and Value-Added Services. If operators to establish a proprietary mobile communication network optimization system, then it is to afford the cost of all the equipment and personnel, so cost is the highest. Once the operators will go out to share resources or shared resources other carriers, then only part of the cost burden on the system. If full use of cloud computing model, the operator has the resources from around the world, the burden of the cost will be reduced to $1 / 10$ or even $1 / 20$.

Large Data Cloud. The basic principle of cloud computing is through the cloud resources, such as large-scale hardware platforms, data centers, providing resources to rent, application hosting, outsourcing and other services to Internet customers. Huge cloud resources, just to meet the mobile communication network needed to optimize data management. The use of cloud computing model, operators can share experience optimization program library, seeking similar cases, greatly simplifies the optimization process. Establish an optimized network optimization program library staff provides optimized basis for the implementation of the communication network.

Lower Maintenance Costs. To ensure the normal communication network optimization smooth running, often need to optimize computer, terminal server, etc. for maintenance, upgrades and updates. Because cloud computing model no limit to the configuration of the user terminal, and 
therefore do not have the relevant skill on the pains associated hardware upgrade, operators of manpower and material resources will be relatively reduced, thereby reducing the communication network optimization costs, greatly improve the optimization effectiveness.

\section{The Communication Network Optimization Model Framework Based on Cloud Computing Model}

Actuality cloud computing and communications network optimization, network optimization framework proposed communication, the purpose is under the influence of cloud computing technology, the study of the communication network optimization deeper thinking. Communication network optimization framework is consisted of communication network optimization environment, identification systems, data storage cloud, cloud resources and personal end devices.

By a communication network to optimize the environment and provide cloud computing services companies to provide network services to telecommunications operators, carriers obtain cloud resources through the network to find the required optimization program, and the premise is to establish a relationship between the company and the network operator. Among them, the identity link is very important, it is assigned a unique username for operators, after obtaining certification and operators can enjoy the service, thereby enhancing the security of the system.

\section{Conclusions}

The mobile communication network optimization framework based on cloud-computing model can be a good solution to the software problems of mobile communication network optimization and simplify network optimization program, while maximizing resource utilization, minimization optimize cost, in line with the business objectives which corporate operators pursue. The cloud computing not only injected fresh blood but also brought challenges to the mobile communication network optimization, but with the matures of cloud computing technology, the cloud computing model has good development prospects in the mobile communication network optimization.

\section{References}

[1] Jian Zhang: Telecom Network Technologies, Vol. 1 (2009) No 53, p.15-18

[2] Liyu Lin: Communication Technology, Vol. 12 (2008) No 27, p.33-38

[3] Rajkumar Buyya: Future Generation Computer Systems, Vol. 6 (2009) No 25, p.599-616

[4] Jieming Liu: Guangxi Normal University, Vol. 3 (2007) No33, p.121-124

[5] Information on http://info.codepub.com/2008/05/info-19352.hml,2008-05-10/2008-08-15. 